\title{
Public Management and the Essential Public Health Functions
}

\author{
Peyvand Khaleghian \\ Europe and Central Asia Region \\ World Bank, 1818 H St. NW, Washington, D.C. 20433 \\ Email: pkhaleghian@worldbank.org \\ Monica Das Gupta \\ Development Research Group \\ World Bank, 1818 H St. NW, Washington, D.C. 20433 \\ Email: mdasgupta@worldbank.org
}

World Bank Policy Research Working Paper 3220, February 2004

The Policy Research Working Paper Series disseminates the findings of work in progress to encourage the exchange of ideas about development issues. An objective of the series is to get the findings out quickly, even if the presentations are less than fully polished. The papers carry the names of the authors and should be cited accordingly. The findings, interpretations, and conclusions expressed in this paper are entirely those of the authors. They do not necessarily represent the view of the World Bank, its Executive Directors, or the countries they represent. Policy Research Working Papers are available online at http:/lecon.worldbank.org.

We are grateful to George Alleyne, Varun Gauri, Jeff Hammer, Sanjay Pradhan, and Shahid Yusuf for comments and suggestions. An earlier version of this paper was prepared as a background paper for the Disease Control Priorities Project of the U.S. National Institutes of Health. 


\section{Abstract}

This paper provides an overview of how different approaches to improving public sector management relate to so-called core or essential public health functions such as disease surveillance, health education, monitoring and evaluation, workforce development, enforcement of public health laws and regulations, public health research, and health policy development. The paper summarizes key themes in the public management literature and draws lessons for their application to these core functions.

\section{Contents}

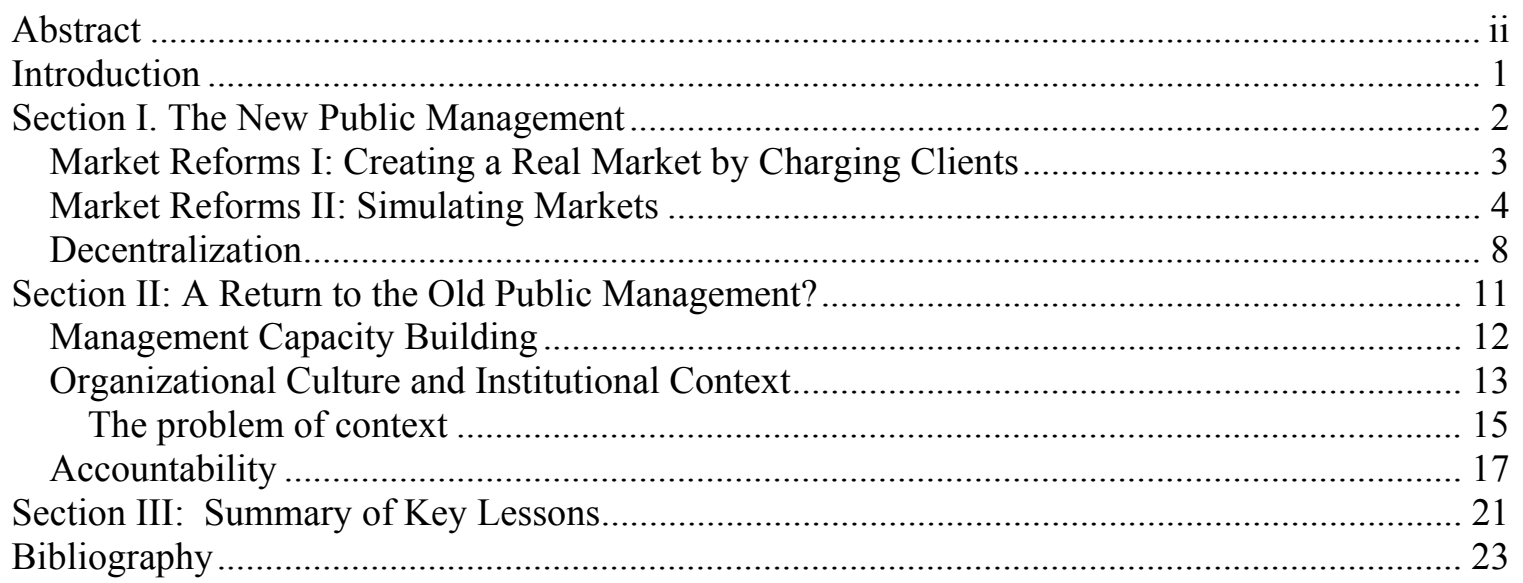




\section{Introduction}

This paper provides an overview of how various approaches to improving public sector management relate to the so-called core or essential public health functions (EPHFs) such as disease surveillance, health education, monitoring and evaluation, workforce development, enforcement of public health laws and regulations, public health research, and health policy development (IOM 1987; PAHO 2002). Its purpose is to summarize key themes in the public management literature and draw lessons for the EPHFs. Section I summarizes "new public management" approaches. Section II reviews traditional approaches to public administration and their relevance to the EPHFs. Section III summarizes lessons in point form.

Two points are essential to understanding the discussion that follows. The first relates to the economic nature of the EPHFs. In economic terms, most EPHFs are public goods. This means that they are non-rival (i.e., consumption by one person doesn't preclude consumption by another) and non-exclusionary (i.e., their benefits accrue to the entire population and can't be restricted to a discrete group). For example: once erected, a health education billboard benefits everyone who views it, no matter how many people do so (i.e., non-rival); and anyone who wants to view it can, given its public location (i.e., non-exclusionary). This is distinct from private goods - e.g., cancer treatment — which, like most commodities, are both rival and exclusionary. Some disease control services fall into a middle category because they have both private and public characteristics. Immunization is one example. While immunization has private benefits for the vaccinated individual, it also has public benefits because of its contribution to herd immunity and the protection of others. The same is true of TB treatment, which benefits the sick individual but also reduces the chances he will infect others-i.e., an external benefit (externality) in addition to the private benefit. These distinctions are not purely academic. They have major implications on how services should be financed and delivered and are fundamental to the discussion below.

The second point relates to the difference between public health services and public health functions. Many traditional public health services-immunization, std clinics, TB control etc.- - are merit goods; the public health functions, however-policymaking, disease surveillance, population health assessment, health education etc.- - are almost all pure public goods. The service-function distinction is not always made clear in the literature but is of considerable practical relevance when it comes to questions of management and financing. Public health services are often relatively easy to measure, e.g., with indicators such as the number of children immunized or the number of TB cases treated. This makes them easier to manage and provides a wider scope for innovations in service delivery compared to public health functions, whose public good nature of complexity of measurement pose special challenges. As such, the public health 
functions are more akin to other "core government functions"- e.g., revenue collection, maintaining law and order-and draw on similar principles for their management. ${ }^{1}$

\section{Section I. The New Public Management}

Until the early 1980s, the public sector in most countries was monopolistic, centralized, hierarchical, inflexible, unresponsive to users and insulated from the private sector and other agencies outside government. With budget crises and the realization of significant inefficiencies in the public sector in the early 1980s, and with the coincident rise in theories and disciplines such as public choice theory, principal-agent theory and transaction cost economics, it was recognized that traditional approaches to public administration were in need of reform. A range of reforms - collectively referred to as the new public management or NPM-rapidly formed a new model of state management. At the heart of these reforms was a shift from government by control to government by contract. This typically involved changes in organizational structure (e.g., moves toward managerial autonomy or corporatization of public entities) and introduction of market processes (e.g., through formal privatization or market-simulating reforms within the public sector, such as purchaser-provider splits and decentralization), and it came to imply a redefinition in the government's role from that of direct service provider to one of stewardship, oversight and regulation (Batley 1999a). The poster-country for these reforms was New Zealand, where sweeping reforms were carried out in the late 1980s - sparing no sector-and which remains as the most comprehensive example of a NPM-motivated reform process to date (Bale and Dale 1998).

The concepts of NPM resonated with health policy specialists and had a major influence on health reforms in the 1980s and 1990s. In most cases the reform effort made no distinction between curative and preventive services and applied similar prescriptions to both. Some concepts - such as purchaser-provider splits, hospital autonomy and decentralization - were effective and took hold for curative services. The greatest success was reported in industrialized countries with high levels of administrative capacity and political stability, such as Singapore and New Zealand, though developing countries such as Ghana, Zimbabwe, Sri Lanka and Thailand also attempted them to various degrees (Russell, Bennett, and Mills 1999). For public health and preventive services, however, impact evaluations - which weren't conducted until the late 1990s - revealed a less positive picture. In this section we examine new public management strategies for public health under three headings: true market reforms, i.e., those involving user charges and provider competition; pseudo-market reforms, e.g., purchaser-provider splits, contracting and other market-simulating reforms; and decentralization.

1 For a more general discussion of issues of public management for service delivery, see the recent World Development Report (World Bank 2003). 


\section{Market Reforms I: Creating a Real Market by Charging Clients}

The negative impact of user fees on preventive services has been abundantly documented. User fees have been shown to cause immunization coverage rates to fall (WHO 1999). The same has happened with other disease control activities. In Nicaragua, for example, reductions in the government budget for malaria control led some areas to adopt user charges for diagnosis and treatment, a practice that had been proscribed since 1947; these in turn led to a fall in the numbers being tested and a consequent rise in malaria incidence (Garfield 1999).

For the EPHFs, user charges are not an option. Consumers will not voluntarily pay for disease surveillance or health education campaigns, given their public good nature. (This explains why tax financing is used to pay for these services.) But in some countries, severe reductions in the budget for public health have forced public health agencies to generate their own revenues by any means possible. Some responded by seeking donor funds to support public health. Others used within-facility cross-subsidies from curative care to public health services, though this was only effective when the two services were jointly managed and when a high level of commitment to public health was present.

In China, however, no such compensations were provided. In the early 1980s China embarked on a radical program of economic reforms. The reform program included introduction of service charges in health facilities to make up for reductions in budgetary support from the government, and a laissez-faire attitude toward competition between providers, including providers at different levels in the service hierarchy. The idea was to improve efficiency by exposing health facilities to competitive market pressures. Reforms were applied to all health agencies, curative and public health alike. By the mid-1990s government budgetary support covered only salaries at health facilities and provided nothing for operating costs. These were supposed to be covered by revenues from the facility itself. The impact of this incentive environment on curative care has been well documented. ${ }^{2}$

A recent study evaluated the impact of these reforms on public health and preventive services (Liu and Mills 2002). It found mixed but generally negative results. Inter-level competition led to a decline in information-sharing and technical assistance between public health agencies at township, district and province levels, with negative consequences for disease surveillance and training opportunities for lower-level staff. The need to generate revenues led to an underprovision of public goods (surveillance, health education, preventive services) and a bias toward high-revenue services such as fee-based health inspections; reduced efficiency by causing duplicate inspections from public health agencies at different levels - each of them motivated by the need to generate revenues - and a general overprovision of inspections; and had negative equity effects because of agencies' tendency to focus on profitable enterprises rather than carrying out inspections across the board. The most significant finding was a fall in the provision

\footnotetext{
${ }^{2}$ Zheng and Hillier 1995; Liu and Hsiao 1995; Bloom and Xingyuan 1997; Bloom 1998, Wang and Cai 2003.
} 
of public preventive services that could not be charged for, including outbreak investigation and response, preventive programs such as immunization and vector control, monitoring of diseases and health status, and health education programs. Since the government budget provided only for salaries, public health agencies had to cross-subsidize provision of these services from revenues generated through profitable activities such as health inspections. Not surprisingly, most agencies did not do so consistently. A MOH report in 1993 found that for non-revenue generating services, more than 80 percent of public health agencies were performing below expected standards; a third were provided at less than half the required level.

\section{Market Reforms II: Simulating Markets}

A second category of NPM-inspired reforms involved the use of market principles within the public sector and the promotion of public-private links. The usual process involves creating a split between purchasing and provision; using output-based contracts for which private and public firms compete (rather than input-based financing of public agencies alone); devolving managerial autonomy; and experimenting with incentive payments and other ways of improving worker productivity. The motivation was to improve efficiency by introducing market competition and reducing the monopoly power of government agencies. We discuss each separately.

Splitting and contracting. "Splitting" refers to the creation of separate agencies to purchase and provide services, the former contracting with the latter on the basis of expected outputs rather than controlling it in a hierarchical way. "Contracting" includes not only publicpublic contracts, as in the case of purchaser-provider splits wholly within the public sector, but also to the use of private firms or providers to deliver government services-sometimes in competition with public providers - through contracts with a purchasing entity. In both cases the idea is to move away from public management via hierarchical control and toward management by contract.

Splitting purchasing from provision has several effects. By separating the two functions, it allows purchaser and provider agencies to each concentrate on their area of comparative advantage; it forces purchasers to be explicit about what they want from service providers; and it helps purchasers hold providers to account. It also allows private companies to compete for public contracts, in principle improving efficiency. Some countries have taken the concept to great lengths. In New Zealand, even the military is divided into a purchaser, the Ministry of Defense, and a provider, the Defense Forces. In this case the motivation was not to introduce competitive pressures, since there is still only one provider, but rather to improve accountability relationships and make explicit the government's expectations of the military (Bale and Dale 1998).

These reforms have also be implemented in developing countries, though not as extensively as originally thought (Russell, Bennett, and Mills 1999). Implementing a government by contract approach requires strong management capacity and good information systems and can 
impose significant administrative costs, particularly for services where measurement is difficult. Contracting is only effective when outputs can be clearly specified and performance clearly measured (Bennett and Mills 1998). Contract specification must be clear, information must be available for monitoring purposes, and an arms-length relationship must exist between government and provider (Batley 1999). While this may be the case for some preventive services - immunization, cold chain management, campaign-based activities or nutrition interventions such as those reported by Marek and others (1999) in Senegal and Madagascar - it is not the case for most EPHFs. Why? First, because measuring performance in the EPHFs is complex, costly and requires strong information systems; and second, because contract management of this sort requires technical and administrative capacities that are weakly developed in many countries.

Functions such as surveillance are so complex and spread-out that monitoring a private contractor would be a costly exercise - however good the indicators chosen - and might lead to principal-agent related efficiency losses such as those observed when hard-to-monitor services are decentralized (Hurley, Birch, and Eyles 1995). Effective monitoring also requires a high level of technical capacity within government, without which contractors will take advantage of the government and fail to be held accountable in classic principal-agent fashion. A common argument for contracting in developing countries is therefore circular: since capacity is low in government, contracting out to the private sector is an option; but for contracting to work well, capacity needs to be high. Given the limited capacity of many developing country governmentseven in the most basic areas of administration such as billing, recording etc. - their ability to write, monitor and enforce contracts for complex services is likely to be very limited indeed. Contracting should therefore be approached with care and not as a substitute for strengthening the capacity of government itself. Given the high transaction and monitoring costs involved, efficiency gains are unlikely. Batley (1999) writes:

Particularly in the social sectors... where government retains critical ultimate responsibilities, the transaction costs of radical reforms which create multiple agencies are likely to outweigh the efficiency gains of "unbundling." More modest ambitions and simpler reforms which avoid the creation of complex, interdependent organizational arrangements are more likely to have beneficial effects.

Managerial autonomy. Essential to effective "management by contract" is giving line managers the autonomy to fulfill their contracts however they see best. Thus, as part of NPM reforms, governments not only drew up contracts for public agencies that specified outputs rather than inputs; they also gave their managers the autonomy to manage inputs and human resources and develop implementation strategies without interference. In some countries this involved legislative changes to place public agencies under corporate law and remove them from civil service rules. 
Given the complexity of contract-based approaches for the EPHFs, is managerial autonomy relevant to these services or not? The answer is a qualified yes - not because of its NPM connections but because of the stifling effect of centralized, command-and-control management on service delivery in general. Governance arrangements in many developing countries provide little if any autonomy to line managers. This has at least two harmful effects: it deprives managers of the freedom to adapt services to local conditions and to manage staff and resources flexibly, and it hinders the development of general management skills in the public sector. Standard rationales for limiting managerial autonomy are to ensure consistent policy implementation and prevent corruption. However, severe restrictions on managerial autonomywhich exist in many countries - frequently go beyond what is necessary to achieve these. This frequently goes unrecognized by central-level managers and policymakers. In India, for example, 80 percent of district health managers said that having more autonomy would help them do their job better, while their superiors at the state level consistently thought they had enough autonomy already (Das Gupta, Khaleghian, and Sarwal 2003). Grindle (1997) points out that a certain degree of autonomy - especially in personnel matters - can help managers develop a positive organizational climate and is consistently associated with good performance. Giving managers the scope to experiment with resource allocation and financing might help ease the problems associated with fiscal centralization, such as the irregularity of transfers and disruptions in paying staff or suppliers (Das Gupta, Khaleghian, and Sarwal 2003). It might also promote innovation, which is otherwise constrained by managers' perception of their superiors' disapproval. But too much autonomy can also be a dangerous thing, leading not only to policy fragmentation and selfserving behavior by managers but also imposing high transaction costs (Batley 1999).

That said, each type of service will have its own balance. For some, such as law enforcement and provider accreditation, where conformity and consistency are essential, managerial autonomy may need to be circumscribed. The same may also be true where public goods or significant externalities are involved, since delegation may lead these to be ignored in the same way as decentralization does (see below). But for others - investigating health hazards, building community health profiles, providing health education and mobilizing community partnerships. among others - moderately widening the scope of managers' authority can promote innovation and improve the adaptation of services to local circumstances without compromising the essential integrity of the function or impeding the need for consistency in core areas (e.g., notifiable diseases, basic reporting systems etc.).

Experience with managerial autonomy is an essential precondition for the introduction of more complex administrative reforms later. But wide-ranging autonomy should not be introduced into public sectors characterized by informality and weak rule-enforcement, since autonomy in these contexts can lead to abuse. As rules and formality are established in the public sector, autonomy can be gradually expanded. As Schick (1998) points out, drawing lessons from the New Zealand model: 
...no country should move directly from an informal public sector [i.e., one in which the deficiency of formal systems is made up by informal behavior, e.g., low pay is compensated by weak monitoring of absenteeism or the taking of informal payments] to one in which managers are accorded enormous discretion to hire and spend as they see fit. New Zealand did not make this leap, and neither should other countries. Before reform New Zealand operated under budgets that controlled spending and corresponded to actual transactions; it also had a civil service system that governed how public employees were hired and paid. In other words, it had a formal public sector. This is an essential precondition for adopting elements of the New Zealand model [i.e., of extensive NPM-inspired managerial reforms].

Improving productivity via performance incentives. A third strategy involves the use of performance-linked pay, bonuses and other financial incentives to influence provider behavior. A large literature exists on provider payment methods in the curative sector, but these methods do not transfer easily to public health services for several reasons. It is easier to measure curative services than public health ones, and incentive systems can therefore be linked to outputs more easily. This is not true of all public health services - immunization, family planning and mass Vitamin A supplementation do have measurable outputs, for example — but for public health functions, the complexity of their measurement makes it difficult to incentivize performance. The use of targets even for measurable public health services is not without problems. False reporting is common and often intensifies when target achievement is linked to incentives (UNICEF 1996). This can push up monitoring costs which in turn can outweigh the expected productivity gains associated with the incentive scheme in the first place: or in the absence of effective monitoring, it can lead staff to redirect their attention away from service delivery and toward data manipulation, thus reducing effectiveness. So while targets are useful for short-term, focused, disease-specific campaigns - and should be used with caution even there - they can be problematic for the more diffuse, long-term action required of the EPHFs. An alternative strategy to measure performance - the use of health outcomes - is also problematic, given the time lags involved and the presence of many confounding variables.

Public health activities also require extensive collaboration between staff-in contrast to curative care, where much depends on the provider's behavior alone - and incentive schemes focused on individuals can therefore have a negative impact on effectiveness by reducing information-sharing and cooperative behavior. The example of China is instructive in this regard. When public health agencies were forced to compete, their willingness to share information and work collaboratively declined — and with it their ability to carry out surveillance, health promotion and other joint activities effectively (Liu and Mills 2002). On a more positive note, Vietnam's recent experience with shared incentives in commune health stations-where bonuses accrued from curative service delivery are shared with public health staff, and where the performance of public health staff reflects on the station's ability to win awards and recognition from central and provincial health authorities - seems to have worked well. (An important distinction is that in Vietnam, public health services receive their operating funds separately from curative services, via direct central grants, and are not cross-subsidized from curative care revenues as they are in China. Without a dependency relationship between the two services, 
cooperative incentives become easier to implement.) Examples from Taiwan and Korea show that group-wide incentives work well for public health staff (Sung and others 2003; Yu and others 2003). In both countries, central health agencies use competitions, non-monetary awards and group recognition to encourage good performance by public health units. These incentives, combined with the respected technical authority of the granting agencies and their reputation for fairness in making awards, have institutionalized efforts to perform well and are weighted toward organizational behaviors conducive to effective public health action-e.g., collaboration, teamwork, information-sharing, etc. - rather than toward focused targets that might have the opposite effect (Khaleghian and Das Gupta 2003). Wade's (1997) account of irrigation workers in Korea provides similar illustrations, as does Tendler and Freedheim's (1994) account of health workers in Céara, Brazil.

Incentives aren't everything, of course (Gauri 2001). However good the incentive scheme, workers can still lose motivation because of other aspects of their work environment. For example, many authors attribute India's shortcomings in public health to its reliance on targets as a way of motivating and measuring health worker behavior (Nair and others 2001). But equally important are public sector practices that limit the flexibility of district health managers to allocate their budgets or reward their staff, reduce worker motivation by subjecting them to erratic disbursements in pay, provide little opportunity for workers' professional development or recognition, enforce rules of conduct unfairly, and make it so difficult to fire poorly performing workers that few incentives remain for workers to perform at their best (Das Gupta, Khaleghian, and Sarwal 2003). Not surprisingly, these lead public health efforts such as immunization, disease surveillance and health promotion - all of which depend heavily on the cooperation and performance of a far-flung workforce- - to fail.

These points lead to three conclusions. The first is that for performance pay to be useful for the EPHFs, measurement indicators need to be chosen carefully. Complex instruments, while accurate, may be unworkable and costly; while instruments that are too simple may increase the likelihood of opportunistic behavior and have other perverse effects. Second, they suggest that performance incentives in public health should be team- or network-based rather than individualized, given the importance of collaboration. And third, they highlight the role that nonfinancial incentives - recognition, training opportunities etc. — can play in encouraging good performance in a sustainable way.

\section{Decentralization}

Decentralization was a common theme in health and public sector reforms in the 1990s and remains this way today. Its proposed benefits center on the fact that it brings decisionmaking processes closer to the community and thereby improves the quality of information available to local decision makers, ensures that local decisions are relevant and adapted to local needs, and improves the ability of the public to hold decision makers and their agents (i.e., public officials) accountable. But the realization of these benefits has not been universal. In some countries, 
decentralization has led to corruption and elite capture because of inadequate checks and balances at the local level. In others, local decision makers - unconstrained because of the lack of accountability mechanisms at the local level - have continued much as they did before, showing greater allegiance to central superiors than to local representatives. In many others, local officials have found themselves without the basic administrative capacity to take on their new roles, leading to failures in service delivery and the basic functions of government. In yet others, decentralization policies have weakened central government agencies to the extent that their corrective hands are tied, leading to policy fragmentation, an inability to take residual responsibility when jurisdictions fail, and an inability to adjust for inter-jurisdictional inequalities.

In addition to these capacity and institutional prerequisites, a further set of conditions is necessary for decentralization to be successful. These relate to the nature of the activity being decentralized. Put simply, activities and services that are "visible" to the public, that have direct, measurable benefits to individuals and for which the public is prepared and able to express wants and preferences tend to fare better under decentralization than others. School management is one example. Since parents have a vested interest in the effectiveness of their schools, and since they are able - even if without perfect accuracy - to judge the quality of the school's activities, devolving the responsibility for schools to a local government can be an effective strategy, as can introducing community co-management in the form of school boards (Jimenez and Sawada 1999, Filmer 2002). But many services do not have these characteristics. This is very much the case with the EPHFs, which are either invisible (surveillance), hard to measure (most EPHFs) or have benefits that are difficult to quantify for individual communities and are therefore neglected by rationally self-interested local authorities (again, most EPHFs). Akin, Hutchinson and Strumpf (2001) summarize this argument as follows:

\footnotetext{
"[Theoretical models predict that] decentralization can lead to an increased government provision of private goods at the expense of public goods. This is because local governments ignore the spillover benefit of public goods to neighboring governments or are less subject to demands of international aid agencies who pressure for public goods projects. But perhaps more importantly, it is because when private type goods are allowed to be chosen, local citizens will behave rationally. They will reveal preferences for goods that most benefit them directly. Thus the argument goes that very decentralized provision of goods by governments, in which local citizens push for choices given a budget constraint, will often lead to a revealed preference problem similar to the one that leads to the necessity for public provision to begin with. Small governments will tend to vote to provide the goods for which citizens reveal preferences. They will therefore tend to spend on private goods type curative care clinic visits rather than on public goods type services such as health education and communicable disease control. Small governments may, therefore, behave too much like individuals, with the residents not revealing their preferences for public goods but in fact for private goods provided publicly. The hypotheses from the model, therefore, suggest that, in contrast to the conventional wisdom, decentralization of allocation decisions for services such as health care may in fact reduce societal welfare."
}

Khaleghian (2003) illustrates this with reference to immunization programs: 
[Another] prediction is that community participation, especially in resource allocation decisions, may have a negative impact on immunization programs. The reasons for this are not difficult to understand. Communities, faced with a multitude of pressing needs and generally unaware of the value of immunization, may consider immunization to be a low priority among the various other goods and services - including curative health services - which their local authorities can provide. In the absence of other incentives (such as strong central pressure or financial controls), local authorities may respond to these preferences by allocating funding away from immunization - and may not in any case be held accountable for its provision by the community, whether directly or through electoral mechanisms - and local immunization programs may suffer as a result. Even with central pressure and oversight, however, the principal-agent relationship between local and central authorities may lead to the same conclusion, with local authorities neglecting central requirements and responding more strongly to local preferences instead.

Published accounts tend to confirm these expectations. In Uganda, local governments spent less on public and semi-public goods after decentralization than before it, with a consequent underprovision of essential public health services such as immunization (Akin, Hutchinson, and Strumpf 2001). In the Philippines, decentralization shifted the balance of health expenditures away from prevention and toward curative care (Solon and others 1999 cited in Soerojo and Wilson 2001). In Nicaragua, local authorities ignored central directives to allocate 46 percent of their health expenditures to primary health care and instead allocated larger shares to secondary care, with immunization coverage in the affected districts falling by up to 50 percent in three years (Birn, Zimmerman, and Garfield 2000). In Indonesia, tight competition from other sectors led local mayors to abandon their commitment that 15 percent of their budgets should go to health (World Bank 2001a, cited in Soerojo and Wilson 2001). Similar phenomena have been noted in Tanzania (Gilson and Mills 1995), Uganda (Jeppson and Okuonzi 2000) and Colombia (Muñoz-Nates 1999, cited in WHO 1999).

This raises a dilemma for the EPHFs. On one hand, many of the EPHFs need local adaptation and tailoring to be most effective, and can not be implemented in a rigid, centrally determined way. This is especially true in large and diverse countries with inter-jurisdictional differences in language, culture or epidemiological profile. On the other hand, decentralizationin the sense of formally transferring the responsibility for these functions to local governmentseems not to work well either. Different approaches have been used to resolve this dilemma. Some countries have retained central management of the EPHFs but established field offices to adapt and tailor them to local needs. This was the prevailing pattern before the 1980s, when the dominant model of decentralization in developing countries was deconcentration to field staff rather than devolution to independent local governments (Manor 1999). The advantage of this approach is that it preserves inter-jurisdictional consistency and prevents local neglect of invisible services (or those that don't bring in more votes); but its disadvantage is that bureaucratic rigidity frequently sets in and local adaptation fails to materialize. The reasons for this frequently center around a lack of managerial autonomy, as described earlier. The other method - which has become more common in recent years as countries have adopted wide-ranging devolutionary policies, sometimes as part of structural adjustment efforts - has been to compensate for local control of the EPHFs by retaining a measure of central influence through financial controls, e.g., grants-in-aid or earmarked funds. This is typically a "second best" solution implemented after the EPHFs have been devolved, because reversal of decentralization policies seldom occurs (Manor 
1999; Dillinger 1994); and it faces a number of implementation problems in developing countries, not least of them being the difficulty faced by central health authorities - themselves reduced in strength and number by decentralization policies that typically reduce the size of central government-in monitoring the activity of local governments. So while formal devolution makes adaptation more likely, it makes central oversight more difficult and in turn can have a negative impact on the effectiveness of surveillance, health promotion and other efforts.

As implied above, the decision on which path to take is seldom a rational one, since decentralization is a political reform motivated principally by non-technical considerations (Smith 1997). The implications for the EPHFs therefore differ according to the path chosen to local political factors, including political factors within the health sector (Collins 1989). Several generic issues do emerge. First, the economic characteristics of the EPHFs make them inherently poor candidates for devolution to local government control, particularly when local governments are weak. Even if this devolution occurs, it should not be accompanied by full fiscal responsibility for these functions, since the incentive environment facing local governmentseven in industrialized countries such as the United States - typically leads to underfinancing of these functions. Second, and irrespective of the path chosen, decentralization policies should not be allowed to reduce the strength or capacity of central government agencies concerned with the EPHFs. Without strong central oversight, whether of deconcentrated field offices or of health officials in local government, the delivery of key public health functions will suffer. Central public health staff serve a number of important functions. In addition to receiving and interpreting information for the benefit of policy makers, they also have an inter-jurisdictional perspective necessary for effective coordination in disease control and play an important role in providing technical assistance and overseeing the activity of health staff at subnational levels (Das Gupta, Khaleghian, and Sarwal 2003). If these functions are compromised - as they very often are in decentralizing reforms, where the size of central government is reduced across the board and Ministries find themselves shrunk to a fraction of their former size - the EPHFs will fare poorly. Third, effective management of EPHFs - whether in a deconcentrated or devolved setup - requires better measurement instruments and attention to management. The management issues have been summarized already and will not be repeated here, except to highlight their special importance in administering a far-flung workforce (as in deconcentration). The measurement issues, however, are critical in both cases, since they condition the ability of central health authorities to effectively monitor, manage and support their subordinates in both hierarchical (deconcentration) or indirect (devolution) forms. In the latter case, the issue of measurement assumes added importance because of its links to central funds and the need to monitor how these funds have been used.

\section{Section II: A Return to the Old Public Management?}

Given the extent of market failures in the health sector-particularly for public health functions - and the limited administrative capacity of governments in most developing countries, the role of NPM-inspired reforms is limited and a return to the "old public management" appears necessary on the surface. This does not imply that public health should be managed in a 
centralized, bureaucratic and unresponsive way. It just means that other approaches to strengthening management need to be found, ones that take into account the economic characteristics of the public health functions and focus on improving their delivery by the government itself. This section summarizes three such approaches: management capacity building; reforms to improve organizational climate; and efforts to improve performance by improving accountability, both hierarchically within government and externally to the public and civil society.

\section{Management Capacity Building}

Russell, Bennett and Mills (1999) highlight the limited capacity of service managers in developing countries and give examples of how even basic administrative tasks - such as recordkeeping and paying suppliers etc.- - are carried out poorly and inconsistently in some countries (Marek and others 1999). Strengthening these capacities is a necessary precondition for improving management: hence, perhaps, the affection of donors for training activities and management-strengthening exercises. But this kind of direct, training-oriented capacity building is not sufficient; changes in the broader administrative context are also necessary. In many countries, unit managers are forced to make repeated visits to superiors in the capital city to gain approval for basic administrative functions such as releasing pay and signing leave forms (Nchinda 2003). This is not an evidence of weak administrative capacity but rather reflects weaknesses in administrative systems and rules - the weakest areas being finance, accounting and human resource management (Russell, Bennett, and Mills 1999) — which are equally important targets for corrective intervention. Similarly, the lack of general management skills is not always a reflection of poor training; it just as often reflects centralized decisionmaking structures and rigid civil service rules that deprive managers of the opportunity to develop these skills, often in spite of numerous donor-sponsored training activities. In sum, if the institutional environment prevents trained managers from exercising their newly developed skills, these managers will either leave the public sector, lose their skills or use them for personal rather than organizational gain (e.g., by covering the tracks of corrupt activity more effectively).

One area of specific relevance to the EPHFs is the ability of managers to collaborate across agency lines (Das Gupta, Khaleghian, and Sarwal 2003). Many public health functions rely on coherent, coordinated action by a number of agencies - surveillance agencies, local governments, water boards, private medical providers etc. - and break down when this collaboration is weak. The ability to collaborate is not an inherent quality of managers; indeed public choice theorists believe that managers actually have the reverse incentive, i.e., to compete with other agencies and expand their own turf. The negative impact of this kind of behavior on public health functions is obvious. Reversing it requires a combination of direct interventions, e.g., sensitizing managers to the importance of collaboration, and institutional changes that facilitate it, e.g., joint strategies, formal collaborative relationships, staff exchanges, and incentive structures that encourage rather than inhibit teamwork (Chen and others 2003, Sung and others 
2003). Experience with these interventions is fairly limited, as most attempts to promote interagency collaboration involve statements of intent but not the institutional changes necessary to back them up.

Batley (1999) points out that many countries, some of them encouraged by technical enthusiasts in the donor community, jumped directly into complex second-generation administrative reforms (e.g., purchaser-provider splits, contracting out etc.) without strengthening administrative skills and general management first. Not surprisingly, these reforms failed. Schick (1998), drawing lessons from New Zealand's reform process, recommends that managementstrengthening efforts in developing countries should involve a series of steps, beginning first with improving the basic elements of public management and strengthening internal controls and only later moving toward more adventurous second-generation reforms. He writes:

\footnotetext{
“...politicians and officials must be able to concentrate on the basic process of public management. They must be able to control inputs before they are called upon to control outputs; they must be able to account for cash before they are asked to account for cost; they must abide by uniform rules before they are authorized to make their own rules; they most operated in integrated, centralized departments before being authorized to go it alone in autonomous agencies.

Once the basics have been mastered, the public sector should be organized according to the principles of internal control. External control and New Zealand-type managerial discretion are not the only options for organizing governmental operations. Internal control is a third possibility."
}

This implies several things for the EPHFs. First, it suggests that training programs for managers will by themselves be insufficient to sustainably improve performance. Second, it shows how complex administrative reforms can only be implemented on a foundation of good general management. And third, it illustrates the substantial effect which institutional contextthat is, the formal and informal rules that govern relationships and behavior of public agents, including rules on recruitment, promotion, managerial autonomy etc. - can have on managers' ability to gain and use their skills. Put simply, efforts to strengthen management of the EPHFs would need to go beyond training to also consider changes in the institutional environment, or if change isn't possible, finding ways to insulate programs from its harmful elements. We next discuss two approaches relevant to the latter point: changes in organizational culture and methods to improve accountability.

\section{Organizational Culture and Institutional Context}

Training efforts are necessary but not sufficient for improving public management. Similarly, public sector reforms focused on pay, staffing levels and government reorganization alone do not succeed in fostering the changes in work attitudes, ethics and organizational culture that are necessary if significant performance improvements are to be realized (Lindauer and Nunberg 1994). Some of these changes are actually antithetical to the work attitudes necessary for effective action, such as when competition is introduced to services such as disease surveillance for which inter-provider cooperation is essential, as has happened in China (Liu and Mills 2002). Grindle (1997) hypothesizes that the "missing link" in public sector reforms is a 
focus on organizational culture. She defines this as "a shared set of norms and behavioral expectations characterizing a corporate identity" and uses case studies from five high-performing public institutions in developing countries to illustrate its dimensions. According to Grindle, the idea that organizational culture can stimulate good performance goes against popular models of bureaucratic behavior. Rational choice and principal-agent models assume self-serving behavior on the part of public officials and their workers and are good at explaining why things go wrong. But they are less effective at explaining why in some organizations, things go right - and why workers in these organizations conform to the public interest model of "principled" agents working in the public interest. As Stiglitz (1999) points out,

The problem is that the wrong lessons have been drawn out of those empirical and case studies showing the failures of government programs in the past. Yes, government programs may not work and they may fail to deliver services to the very poor, but this does not necessarily mean that government programs cannot work. The objective should have been to inquire into what caused the failures and to ask how those failures can be averted. Only if one could be convinced that there was something inherent about public provision that made failure inevitable — and that this failure was worse than the market failures being addressed - should one lose hope. Too often, the public failures can be easily traced to inadequate incentives; governments need to recognize that incentives apply to them too, and not just to markets.

Grindle (1997) identifies four factors consistently associated with good performance in public organizations: a sense of organizational mission, defined as a widely held sense of purpose within the organization; management styles that encourage participation, flexibility, teamwork, problem-solving and equity; clear performance expectations for staff; and managerial autonomy, particularly in personnel matters.

Organizational mission and mystique. Organizational "mystique" arises when workers feel that they acquire prestige and a good reputation by working for their organization, and when their organization has a reputation for competence, respect and social contribution. Health staff in Bolivia reported a high degree of pride in their organization and a personal commitment to maintaining its good reputation by contributing to the quality of services delivered (Grindle 1997). Key elements of this "mission and mystique" factor included a strong sense of service among staff; identification with norms and values that were thought to have universal validity such as honesty and political non-involvement; a sense that the organization and its employees were somehow unique, whether in their practice or the nature of their mission; a sense that staff selection was based on competence or skill (and thus, a sense of pride in being part of the organization); and absorption of the organization's mission by staff, making it a personal mission as well as an organizational one. These factors were found to be independent of salary scales or other remuneration. Promoting the emergence of these factors requires committed leadership and explicit action, since they seldom arise de novo. In an example from Brazil, Tendler and Freedheim (1994) summarize the use of public information campaigns by provincial government to improve the public perception and status of first-level health workers. The campaigns had a twofold purpose: they helped health workers feel good about their jobs by conveying a positive impression to the community, and they also helped overcome resistance and political capture by 
mid-level governments - in this case local mayors — by publicizing the higher-level government's support of their mission (Das Gupta, Grandvoinnet, and Romani 2004).

Good management. Grindle (1997) identifies four managerial characteristics that are correlates of good performance. These included open and non-hierarchical interactions between managers and staff; managers who insulate their staff from perverse elements of the institutional environment (e.g., rigid civil service rules, political interference or demands for patronage); managers' use of incentives and rewards in a consistent, fair way (even those as simple as "employee of the month" recognitions); and managers who encourage teamwork among staff. Fairness in the application of rules and distribution of material and non-material rewards is the critical factor here.

Performance expectations. Organizations get the best out of staff when staff realize that performance matters. In many developing countries this message is hard to get across; civil service rules or political patronage lead to promotions based on seniority or political connections rather than merit or performance, and workers therefore have little incentive to perform well. But some managers and organizations find ways around this, whether by enforcing strict selection criteria (such as examinations or multiple interviews) to impress on candidates the importance of quality and performance; using induction periods to communicate organizational norms and performance expectations; having clear job descriptions and clear and fairly enforced rules about remuneration and promotion; or using probationary periods, time-limited contracts and performance reviews linked to clear reward and punishment actions even when these actions are not part of general civil service norms. The key message to workers should be that performance matters, and that good performance will be rewarded - even if only through recognition or other non-pecuniary means - and poor performance punished.

Managerial autonomy, particularly in personnel matters. For an organization to rise above public sector norms, managers need at least some autonomy in personnel matters. Managers need the autonomy to advertise and fill vacant positions, promote people on the basis of merit and punish those who do not meet performance expectations. This basic autonomy is lacking in many developing countries, such as India (Das Gupta, Khaleghian, and Sarwal 2003). Granting managers discretion over these decisions does not always led to better performance-it may actually have the reverse effect if managers use their newly gained authority for private benefit — but it does appear to be an important precondition for performance improvement. These issues have been discussed already and are not revisited here.

\section{The problem of context}

Focusing on organizational culture alone will not address the problems of a hostile work context. Just as workers aren't motivated by pay changes alone, they may also fail to respond to changes in the organizational micro-climate if the external environment-what Grindle calls the "action environment" and others call the institutional context - provides significant barriers to action. These problems are pervasive in developing countries and are frequently outside the direct 
influence of sectoral staff. Civil service rules, procedures and decisions are centralized and rigid, limiting innovation and the ability of managers to manage their staff and programs effectively; promotions are based on seniority rather than merit; and political interference is common.

Russell, Bennett, and Mills (1997), surveying the effectiveness of health management reforms in five developing countries, draw similar conclusions about the impeding effect of hostile organizational climate on managerial change. They point out the difficulty of changing this climate given the entrenched nature of existing institutions and the influence of vested interests within the public bureaucracy (p. 773). Grindle argues that a focus on organizational culture should not substitute for efforts to improve the broader institutional environment. She writes:

\begin{abstract}
Encouraging the development of characteristics associated with positive organizational cultures may be an important way of improving public sector performance where the broader economic, social and political environment as well as the public sector in general are seriously detrimental to good performance. Clearly, building performance-oriented organizational cultures is not a substitute for civil service reform or for changes in the institutional context within which public sectors operate in developing countries.... however, promoting organizational characteristics that promote positive cultures may be the "missing ingredient" in the disappointing results of many civil service reforms. (Grindle 1997, p.491)
\end{abstract}

However, she further argues that change is possible in such contexts, provided a minimum of administrative autonomy is given to public managers and is used to good effect.

In response to these problems, some countries have experimented by delegating functions to parastatal agencies. For example in India, some disease control programs have been delegated to semi-autonomous agencies that operate with public money but outside the formal public sector. The purpose of these reforms was to unburden program managers from civil service rules, expand their autonomy in hiring staff, allocating resources and tailoring programs to local needs, and to introduce community oversight by appointing external members to agency boards. Experience with these "disease control societies" has been mixed. In some settings autonomy has been exercised responsibly and programs have become flexible, effective and locally adapted. In others, it has led to more rent-seeking in procurement and a change in the distribution but not the extent of patronage. Establishing autonomous agencies can also undermine the coherence of government action more broadly as well (Das Gupta, Khaleghian, and Sarwal 2003). These agencies can destabilize budgets in three ways: first, by creating a constituency for earmarked funds that undermines the government's ability to reallocate resources as priorities change; second, by creating so-called bleeding stump scenarios where autonomous agencies hold the government to ransom by threatening the unthinkable - e.g., health workers on strike or outbreaks ignored - unless additional resources are provided; and third, by creating liabilities for the government if agencies borrow against state assets. They can also be used to appease fractious coalition members in politically unstable environments, defeating their basic purpose to improve service delivery. 


\section{Accountability}

In the final analysis, the effectiveness of service delivery depends on the extent to which those who deliver it are held accountable for their performance. In this section we consider two kinds of accountability: hierarchical accountability, which is based on bureaucratic controls within government ${ }^{3}$; and social accountability, which involves communities in management and monitoring of programs (Das Gupta and Khaleghian forthcoming).

Hierarchical accountability. This involves monitoring the performance of public servants, rewarding them for good performance and punishing them when performance is bad. This is easier for some services than others. For services where output is easy to measure - the number of bricks laid or amount of taxes collected - this type of accountability is relatively straightforward. Tax collection reforms in Brazil, Mexico and Ghana bear out this point. In all three countries tax collectors were given a pecuniary incentive linked to their productivity in collecting taxes, as a result of which collection rates improved dramatically. In other countries, pay reforms were used rather than monitoring, the intention here being to reduce corruption by "raising the stakes" due to the higher wages which corrupt officials would forfeit if caught. The Becker-Stigler theory postulates that these two approaches are interactive, with higher pay and higher monitoring intensity both contributing to better performance and reduced corruption (Becker and Stigler 1974). Studies of corruption and government performance in several contexts confirm the validity of this hypothesis (Mookherjee 2001).

When measurement is difficult, however, it becomes considerably harder for supervisors to monitor and incentivize staff performance - and correspondingly easier for staff to manipulate the system in their favor-leading in turn to principal-agent problems and accountability failures. This applies to several of the EPHFs. Surveillance, for example, becomes less visible the more effectively it is carried out. Without strong internal controls and process measures, the first sign of a failing surveillance system is frequently a major outbreak - too late for corrective action. The same is true of health promotion. Since the impact of these activities is difficult and expensive to measure, evaluations - when they are carried, which they frequently aren't — are seldom linked back to the staff involved in preparing the program in the first place. As a result these staff operate in an accountability vacuum and have little incentive to "get it right". Monitoring can also be difficult for other reasons. In the case of health inspection, problems can arise when inspectors are expected to survey a large area and there are few supervisors above them. In this context, inspectors know that the likelihood of a supervisory check - i.e., the intensity of monitoring - is low, and they therefore have little incentive to perform efficiently or honestly, since the likelihood of being caught or punished is also low.

These points have important implications for the use of bonus payments and other ways of rewarding good performance. If measurement is weak or imprecise, whether because the service in question is hard to monitor or because monitoring is done poorly, it becomes difficult

\footnotetext{
${ }^{3}$ See the citation from Schick (1998) above.
} 
for managers to identify good performance and reward it accordingly. In India, for example, malaria workers were offered a monetary incentive linked to the number of slides they processed. Since monitoring was weak, workers created artificial smears using dog's blood and used these to claim the bonus (Das Gupta and Khaleghian 2002). Mookherjee (2001) writes:

Even deeper problems arise with respect to defining measures of good performance, conditional on which bonuses can be paid. How does one measure the output of a teacher, or a policeman? [Designing] such performance metrics is tricky even in the context of developed countries, owing to their multidimensional nature, where some dimensions more difficult than others to measure. Measuring and rewarding the more measurable dimensions may be dysfunctional, since they may cause the official to divert effort towards those dimensions that are measured, at the expense of those that are not. For instance, if teachers are evaluated are evaluated on the basis of their students' grades, they might then be motivated to redirect their teaching to emphasize examination technique at the expense of real learning; in extreme cases even to leak exam questions to their students.

This underscores the complexity and danger of using personalized incentives as a way to improve agency performance. (The impact of such incentives on cooperative behavior is also relevant, as discussed earlier.) It also highlights the fact that choices about monitoring are the product of two countervailing forces: the desire for simplicity, which makes measurement easier and cheaper but runs the risk of creating perverse incentives, and the desire for comprehensiveness, which creates more accurate incentives but can be costly to implement.

Here too the institutional context matters. As Mookherjee (2001) writes:

The difficulty of investigating and establishing the incidence of corruption is compounded by civil service norms and legal difficulties in securing dismissal. The worst consequence for a tarnished official is frequently transfer to a less lucrative assignment, or a temporary suspension for a few months. Transfer policies often tend to be used instead by political superiors to reward or punish compliance with their own personal agendas, rather than merit based performance.

Specifically, in settings where political corruption is widespread, monitoring rules are set by politicians who have a vested interest in keeping them weak. Shirley and Xu use data from 12 countries to show how this leads performance-based incentive schemes to fail:

...the governments negotiating our sample contracts chose to pledge actions that they were not motivated or able to implement. Second, with the exception of India, Korea and Mexico, the supervisory agencies were not given the capacity to negotiate, monitor and evaluate well. Their information advantage was reinforced by other government actions, such as giving them low pay and status, frequently moving them within the government, and failing to force the SOEs [state-owned enterprises] to comply with their information requirements. This allowed managers to manipulate the targets, which proved to be a serious failing of all the contracts in our sample.

What do these points imply for the EPHFs? First, they show that monitoring matters. Second, they suggest that monitoring should not focus on outcomes alone, given their distal and distant nature, but should instead use carefully chosen proximate measures that can be used to monitor performance but are still relevant to the production of outcomes. Third, they highlight the potential role of information systems - both HIS and MIS - in providing data inputs for service 
monitoring. And fourth, they imply that for public health functions and services, broadly based performance measures linked to group-wide incentives are probably more effective than highly specific measures linked to individual ones.

Social accountability. Another approach is to make service providers accountable to their constituents, either by having community members participate in managing the service or through providing them with information, choice, or opportunities to voice their concerns if the service fails to meet their expectations. Examples can be divided into interventions dominated by community co-management, choice, information, or "voice" mechanisms.

Community co-management has been a favorite of primary health care enthusiasts since the Alma-Ata conference in 1978, where the notion of community participation was given prominence. Examples abound: for example, the role of communities in managing service during the civil conflict in Panama (Taylor and Taylor-Ide 2000), the emerging role of Panchayati Raj Institutions in overseeing health services in India (Das Gupta, Khaleghian, and Sarwal 2003), the important role of communities in Bamako Initiative countries, and the use of community boards to oversee public hospitals in some industrialized countries (Hurley, Birch, and Eyles 1995; McKee and Healy 2000). Extensive examples can also be found in the education literature, where community co-management of schools has received much attention (Mookherjee 2001). The basic idea is that community input will improve the quality and local tailoring of services and make sure the providers do their job properly. A teacher will find it harder to shirk if community members are, in effect, his bosses; likewise a health worker may take a less heavy handed approach to her clients if they are involved in decisions regarding pay or promotion. Like political decentralization, these approaches are relevant for services where there is a high level of community demand - people want a doctor in the clinic and teachers in the local school—and monitoring is straightforward. It is less relevant for "invisible" services such as the EPHFs, in which communities tend to be less interested because of their public goods characteristics and for which continuous monitoring is not a simple process. The arguments here are the same as those for decentralization, as summarized earlier.

The second approach is to expand the range of providers and give consumers a choice regarding whom they use: in other words, to create a market for the service. Primary curative care is one area where this strategy is commonly used; another is the use of vouchers for primary education, both of which are intended to put competitive pressure on providers (doctors, schools) and thus improve their quality and responsiveness to client demands. For reasons explained earlier, choice mechanisms - and related marketizing reforms - are a poor fit for services that have public goods characteristics or significant externalities. They are therefore of little benefit to the EPHFs and can actually be quite harmful, as the earlier discussion and the China example of Liu and Mills (2002) so clearly show.

The third approach is to disseminate information about performance or expected standards of service delivery. These range from notices on a clinic door to information on test scores or procurement prices for basic drugs. In a well-known example in Uganda, a Public 
Expenditure Tracking Survey revealed that 87 percent of school funds were "leaking"-i.e., disappearing - between central government and schools, either into other programs or the pockets of corrupt district officials. In response, the government started to publicize the amount of monthly transfers to each district in the newspaper and over the radio, and required primary schools to post notices on all inflows of funds. A year after starting the program, leakage had fallen to 10 percent as district officials and school administrators found themselves under greater scrutiny by the public and the media (Reinikka and Svensson 2001). A similar campaign was waged by the government of Buenos Aires, Argentina, in response to pharmaceutical price escalations that were thought to be a result of corruption in procurement. In this case, the fact that the government was collecting information was by itself sufficient to cause prices to fallpresumably because corrupt officials assumed the data collection would be followed by some sort of crackdown (Di Tella and Savedoff 2002). Information-based approaches do not have to be government driven. A free press and civil society can also serve similar functions, as Samuel Paul's report cards on public services in Bangalore show (Paul 1998). Here, a NGO collected and disseminated information on the performance of public utilities and health providers in urban Bangalore. Some improvements in service delivery were reported to follow, though these were more prominent in services such as water and power where private demand is high. The media also tends to focus on such services. As a result, while outbreaks receive plenty of attention, routine service delivery in public health - the day-to-day functions of surveillance, reporting, data analysis - receives no attention at all. In India, democracy and a free press help ensure outbreak control but not routine disease control, analogous to Sen's (2000) analysis that they have been effective in preventing famines, but remarkably ineffective in preventing chronic malnutrition (Das Gupta, Khaleghian, and Sarwal 2003). Thus, relying on informational strategies or the media to hold providers accountable for "invisible" services is not, by itself, a complete solution.

The fourth approach is to provide channels for users to voice their concerns and preferences regarding the service. Client surveys are one such approach. In these, client feedback to the provider is used to influence service delivery. Another approach is the use of patient advocates or other independent bodies to which service users have access and which have formal influence with providers. Like the other approaches summarized above, voice channels are most effective for private, measurable services such as water supply, irrigation, education and primary curative care. For the EPHFs, they are unlikely to work well because people have little direct interest in the service - except when it breaks down, when the only accountability that remains is ex post and therefore too late - and because measurement is complicated by the nature of the services and their lack of visibility.

These four approaches are interactive and mutually reinforcing. Information can't improve service delivery unless accompanied by some recourse method, whether it be control, choice or voice; and no amount of control, choice or voice is useful unless service users have reliable, accurate and easily interpreted information about the service they are supposed to monitor. These linkages are frequently ignored. In India, the government publishes a service 
charter that promises a set of minimum standards from government service delivery agencies. But until recently the charter provided no information on what people could do if these standards aren't being met, rendering the exercise futile and giving service providers no additional incentive to perform (Government of India 2002). The same is also true of simpler strategies: for example, a notice of opening hours on a clinic door means nothing unless other methods exist for service users to call the doctor to account should he fail to show up.

Context is also relevant. If a community is invited to manage, say, water or irrigation services, it needs the authority to do so effectively. But this authority is not always provided, and community boards find themselves delegated with administrative functions but constrained by civil service rules, patronage structures or other impediments to the real exercise of control. The same could be said of choice, voice and information-based interventions, all of which can be compromised by institutional contexts that "stack the deck" in favor of poorly performing agencies and their staff.

What can be learned from these strategies for the EPHFs? On the surface it would seem the answer is nothing. All of them seem to work best for services where public demand and interest is high, which is not the case with most of the EPHFs. With adaptation, however, the basic principles of social accountability can indeed be applied (with the possible exception of choice, for reasons described earlier). While accountability to the public may not be effective, accountability to civil society organizations with an interest in public health can. This would require the presence of such groups in society - whether they be NGOs, academics or others - or efforts to create and support them, whether government- or donor-sponsored. It would also require institutional changes to allow the exercise of these practices, such as freedom of information laws, forums for non-government inputs to be heard and regulations mandating a timely government response. For social accountability to work for the EPHFs therefore requires two steps: establishing a constituency for the functions themselves, most likely comprised of academics but possibly drawing on interested others as well; and establishing institutional structures to release and disseminate information, allow the exercise of voice, or formalize comanagement relations in a way that allows accountability to emerge. This represents a fruitful area for policy experimentation and an important complement to the more direct methods of improving performance described earlier.

\section{Section III: Summary of Key Lessons}

- Curative services, preventive services and essential public health functions have important distinctions that make it impossible for policy prescriptions and organizational forms from one to be applied to the others without substantial adaptation.

- User fees are not an option for the EPHFs because of their public goods characteristics.

- Promoting competition among agencies responsible for public health functions does not improve efficiency; on the contrary, it impedes collaboration and technical assistance and can therefore compromise the effectiveness of activities such as surveillance and health 
promotion. Organizational reforms that rely on provider competition (such as purchaserprovider splits) are therefore not applicable to the EPHFs.

- Contracting works for some services but not others. For preventive services that are measurable and discrete, such as immunization or campaign-based programs, contracting can be an effective approach-provided there is sufficient government capacity to manage the contracts effectively. But for the EPHFs, where measurement is complex, expensive and requires strong information systems, contracting imposes transaction and monitoring costs that make efficiency gains unlikely and reduce effectiveness.

- Managerial autonomy is important for the EPHFs as a way of promoting adaptation and innovation. It should be introduced cautiously to avoid abuse, but not so cautiously that it fails to materialize at all; but it should also be balanced with instruments to ensure an appropriate degree of policy and program consistency across units and jurisdictions.

- Decentralizing the EPHFs is a risky strategy, since local governments have little incentive to invest in public goods and systematically neglect them. The EPHFs should either remain under central control — with managerial autonomy or other strategies to permit local adaptation and responsiveness - or, if already decentralized, should be subjected to alternative forms of central oversight and control such as grants-in-aid, earmarked funds etc.

- Decentralization should not be allowed to compromise the strength of central agencies responsible for the EPHFs. This often happens as central governments downsize across the board, but it can have a strongly negative impact on the EPHFs given the importance of central coordination, oversight and technical assistance to these functions.

- The institutional environment-i.e., the formal and informal rules and norms at work in government and society at large - are an influential determinant of government effectiveness, including for the EPHFs.

- Efforts to build management capacity through training are helpful but not sufficient to improve managerial effectiveness for the EPHFs, since the latter is heavily influenced by rules and norms in the broader institutional environment that can't be addressed through training alone.

- Public sector norms and rules that impede effective administration should be changed where possible. Since these rules are frequently outside the direct influence of sectoral officials, alternatives - such as insulating programs from these norms and rules or promoting organizational cultures and accountability arrangements that achieve this indirectly - should be pursued instead.

- Provider incentives are complex and difficult to design for the EPHFs and can not be simply transferred from the experience with curative care. For incentives to be useful, measurement indicators need to be chosen carefully: complex instruments may be unworkable and costly, while instruments that are too simple may increase the likelihood of opportunistic behavior. Incentives, where used, should be team- or network-based rather than individualized and should not neglect the role of non-financial benefits.

- Performance improvement can also be achieved in more traditional ways, e.g., by having fairly implemented merit-based selection and promotion criteria, clear job descriptions etc. These should typically be implemented before the adoption of complicated performance incentive schemes and may be sufficient by themselves.

- Strengthening hierarchical accountability within the public health system is essential to strengthening the EPHFs. This requires changes in the capacity, autonomy and behavior of 
service managers (see above) but also requires monitoring systems and instruments that are weakly developed at present.

- Monitoring is critical but instruments to monitor the EPHFs in an operational way are weakly developed at present. Instruments need to strike the right balance between simplicity and complexity and should be designed for operational rather than research use. Outcome measures are not useful for month-to-month program management which requires more proximate indicators. Information systems - both HIS and MIS - can provide data inputs for this kind of monitoring but are frequently too weak to do so.

- Standard information and voice-based strategies do not work particularly well for the EPHFs but can be adapted to do so. This would involve constituency building for the EPHFs and the use of academic, civil society and media groups to monitor and have input to decisions concerning them, rather than using private citizens to do so.

\section{Bibliography}

The word "processed" describes informally reproduced works that may not be commonly available through library systems.

Akin, J., P. Hutchinson, K. Strumpf. 2001. "Decentralized and government provision of public goods: the public health sector in Uganda." Draft paper, MEASURE Evaluation: Carolina Population Center, University of North Carolina and Chapel Hill, March 2001.

Bale, Malcolm, and Tony Dale. 1998. Public sector reform in New Zealand and its relevance to developing countries. World Bank Research Observer 13(1): 103-21.

Batley, Richard. 1999a. The new public management in developing countries: introduction. Journal of International Development 11: 755-60.

Batley, Richard. 1999b. The new public management in developing countries: implications for policy and organizational reform. Journal of International Development 11: 761-65.

Becker, Gary, and George Stigler. 1974. Law enforcement, malfeasance and compensation of enforcers. Journal of Law and Economics III(1): 6.

Bennett, Sara, and Ann Mills. 1998. Government capacity to contract: health sector experience and lessons, Public Administration and Development 18(4): 307-26.

Bloom, Gerald, and Gu Xingyuan. 1997. Health sector reform: lessons from China. Social Science \& Medicine 45(3): 351-60.

Bloom, Gerald. 1998. Primary health care meets the market in China and Vietnam. Health Policy 44: 233-52.

Chen, Paul C.Y., Lai Kah Lee, and Kai Lit Phua. 2003. Case study of Malaysia. In Monica Das Gupta and Peyvand Khaleghian, eds., Public Health Systems and Communicable Disease Control: case studies from Asia, Latin America and Africa. Forthcoming.

Collins C. 1989. Decentralization and the need for political and critical analysis. Health Policy and Planning 4(2): 168-71.

Das Gupta, Monica. and Peyvand Khaleghian. 2002. Field notes from Karnataka. Development Research Group, World Bank, Washington, D.C. Processed.

Das Gupta, Monica, Peyvand Khaleghian, and Rakesh Sarwal. 2003. Governance and disease control: lessons from India. Policy Research Working Paper 3100. Development Research Group, World Bank, Washington, D.C. 
Das Gupta, Monica, Helene Grandvoinnet, and Mattia Romani. 2004. State-community synergies in community-driven development. Journal of Development Studies 40 (3): 27-58.

Das Gupta, Monica, and Peyvand Khaleghian. Overview. In M.Das Gupta and P.Khaleghian, eds., Public Health Systems and Communicable Disease Control: case studies from Asia, Latin America and Africa. Forthcoming.

Di Tella, Rafael, and William Savedoff. 2002. Diagnosis corruption: fraud in Latin America's Hospitals. Washington, D.C.: Inter-American Development Bank.

Dillinger W. 1994. Decentralization and its implications for urban service delivery. Washington, D.C.: UNDP/UNCSH/World Bank Urban Management Programme. Cited in POLICY Project. 2001. "Health Reform, Decentralization, and Participation in Latin America: Protecting Sexual and Reproductive Health." Washington, DC: The Futures Group International, POLICY Project, April 2001

Filmer, Deon. 2002. "Autonomy, Participation, and Learning in Argentine Schools: Findings and Their Implications for Decentralization. Policy Research Working Paper 2766 (January). Development Research Group, World Bank, Washington, D.C.

Garfield R. 1999. Malaria control in Nicaragua: social and political influences on disease transmission and control activities. Lancet 354 (July 31, 1999): 414-18 .

Gauri, Varun. 2001. Are incentives everything?: payment mechanisms for health care providers in developing countries, Policy Research Working Paper 2624. Development Research Group, World Bank, Washington, D.C.

Gilson, L., and A. Mills. 1995. Health sector reform in sub-Sahara Africa. Health Policy and Planning 32: 215-43.

Government of India. 2002. Citizen's Charter Website. Accessed at http://goicharters.nic.in/ on November 15, 2002.

Grindle, Merilee. 1997. Divergent cultures: when public organizations perform well in developing countries. World Development 25(4): 481-95.

Hurley, J, S. Birch, and J. Eyles. 1995. Geographically-decentralized planning and management in health care: some informational issues and their implications for efficiency. Social Science and Medicine 41(1): 3-11.

Institute of Medicine. 1987. The Future of Public Health. Washington, D.C.: National Academy Press.

Jeppsson, A., and S. A. Okuonzi. 2000. Vertical or holistic decentralization of the health sector? Experiences from Zambia and Uganda. International Journal of Health Planning and Management 15: 273-89.

Jimenez, Emmanuel, and Yasuyuki Sawada. 1999. Do Community-Managed Schools Work? An evaluation of El Salvador's EDUCO program. World Bank Economic Review 13(3): 41541.

Khaleghian, Peyvand. 2003. Decentralization and public services: the case of immunization. Social Science \& Medicine, forthcoming.

Khaleghian, Peyvand, and Monica Das Gupta. 2003. The morning after: What to do about public health services after a heady night of passionate decentralizing. Development Research Group, World Bank, Washington, D.C. Processed. 
Lindauer, David, and Barbara Nunberg, eds.. 1994. Rehabilitating government: pay and employment reform in Africa. Washington, D.C.: World Bank

Liu, Xingzhu, and Anne Mills. 2002. Financing reform of public health services in China: lessons for other nations. Social Science \& Medicine 54: 1691-98.

Liu, Xingzhu and William Hsaio. 1995. The cost escalation of social health insurance plans in China: its implications for public policy. Social Science \& Medicine 41(8): 1095-1101.

Manor J. 1999. The political economy of democratic decentralization. Washington, D.C.: Directions in Development Series, The World Bank

Marek, Tonia, Issakha Diallo, Biram Ndiaye and Jean Rakotosalama. 1999. Successful contracting of prevention services: fighting malnutrition in Senegal and Madagascar. Health Policy and Planning 14(4): 382-89.

McKee, Martin, and Judith Healy. 2000. The role of the hospital in a changing environment. Bulletin of the World Health Organization 78(6): 803-10.

Mookherjee, Dilip. 2001. Combating the crisis in government accountability: a review of recent international experience. R.C. Dutt Memorial Lectures, Center for Studies in Social Science, Calcutta

Nair, V.M, K.R. Thankappan, P.S.Sarma, and R.S.Vasan. 2001. Changing roles of grass-roots health workers in Kerala, India. Health Policy and Planning 16(2): 171-79.

Nchinda, Thomas C. 2003. Case study of Cameroon. In Monica Das Gupta and Peyvand Khaleghian, eds., Public Health Systems and Communicable Disease Control: case studies from Asia, Latin America and Africa. Forthcoming.

Pan American Health Organization (PAHO). 2002. Public health in the Americas: conceptual renewal, performance assessment and bases for action. Washington, D.C.: Pan American Health Organization.

Paul, Samuel. 1998. Making voice work: the report card on Bangalore's public services. Policy Research Working Paper 1921. Development Research Group, World Bank, Washington, D.C.

Reich, Michael M. 2002. Reshaping the state from above, from within, from below: implications for public health. Social Science \& Medicine 54: 1669-75.

Reinikka, Ritva, and Jakob Svensson. 2001. Explaining leakage of public funds. Policy Research Working Paper 2709. Development Research Group, World Bank, Washington, D.C.

Russell, Steven, Sara Bennett and Anne Mills. 1999. Reforming the health sector: towards a new healthy public management. Journal of International Development 11: 767-75.

Shaw, R. Paul. 1999. New trends in public sector management in health: applications in developed and developing countries. World Bank Institute Working Paper, Washington, D.C.

Schick, Allen. 1998. Why most developing countries should not try New Zealand reforms. World Bank Research Observer 13(1): 123-31.

Sen, Amartya. 2000. Development as freedom. New York: Alfred A. Knopf

Smith, B.C. 1997. The decentralization of health care in developing countries: organizational options. Public Administration and Development 17: 399-412. 
Soerojo, W., and N. Wilson. 2001. "Decentralization and immunization services in Indonesia." Consultant report prepared for East Asia and Pacific Division, World Bank, Washington, D.C. Processed.

Stiglitz, Joseph. 1999. Incentives in the health sector. Keynote address, International Health Economics Association Annual Meetings, Rotterdam

Sung, Fung-Chang, Yu-Chen Hsu, Chin-Shui Shih, Yaw-Tang Shih, Shiing-Jer Twu. 2003. Case study of Taiwan. In Monica Das Gupta and Peyvand Khaleghian, eds., Public Health Systems and Communicable Disease Control: case studies from Asia, Latin America and Africa. Forthcoming.

Taylor, C.E., and D. Taylor-Ide. 2000. Just and Lasting Change: When Communities Own their Futures. Baltimore, Md.: Johns Hopkins University Press

Tendler, J., and S. Freedheim. 1994. Trust in a rent-seeking world: health and government transformed in Northeast Brazil. World Development 22(12): 1771-91.

UNICEF. 1996. Sustainability of achievements: Lessons learned from Universal Child Immunization. Evaluation and Research Report, January 1996. UNICEF, New York, NY.

Wade, Robert. 1997. How infrastructure agencies motivate staff: Canal irrigation in India and the Republic of Korea. In Ashoka Mody, ed., Infrastructure Strategies in East Asia. Economic Development Institute. Washington, D.C.: World Bank.

Wang, Hong, and Xiaodong Cai. 2003. Case study of China. In Monica Das Gupta and Peyvand Khaleghian, eds., Public Health Systems and Communicable Disease Control: case studies from Asia, Latin America and Africa. Forthcoming.

WHO. 1999. Report of a meeting on health sector reform and priority health interventions: the case of immunization services, Washington, 15-16 November 1999 (WHO/V and B/00.39). Geneva: World Health Organization, November 1999.

World Bank. 2003. World Development Report 2004: Making Services Work for Poor People. Washington, D.C.: The World Bank.

Yu, Seung-Hum, Sang Gyu Lee, Kang-Hee Lee, Jung In Kim. 2003. Case study of The Republic of Korea. In Monica Das Gupta and Peyvand Khaleghian, eds., Public Health Systems and Communicable Disease Control: case studies from Asia, Latin America and Africa. Forthcoming.

Zheng, X., and S. Hillier. 1995. The reforms of the Chinese health care system: county level changes: the Jiangxi study. Social Science \& Medicine 41(8): 1057-64. 